\title{
Impact upon clinical outcomes of translation of PNA FISH-generated laboratory data from the clinical microbiology bench to bedside in real time
}

\author{
Tam Ly \\ Jyoti Gulia \\ Vasilios Pyrgos \\ Masashi Waga \\ Shmuel Shoham \\ Section of Infectious Diseases, \\ Washington Hospital Center, \\ Washington DC, USA
}

Correspondence: Shmuel Shoham

Washington Hospital Center, Section of Infectious Diseases, I 10 Irving Street NW, 2A56, Washington DC, 20010, USA $\mathrm{Tel}+\mathrm{I} 2028777164$

Email shmuel.shoham@medstar.net

\begin{abstract}
Fluorescence in situ hybridization using peptide nucleic acid probes (PNA-FISH) differentiates Staphylococcus aureus from other Gram-positive-cocci in clusters (GPCC). 101/202 patients with GPCC+ blood cultures were randomly assigned to clinician-notification of PNA FISH results. Notification was associated with reduced mortality ( $8 \% \mathrm{vs.17 \%}$, $p=0.05$ ), further antibiotic use (median -2.5 days, $p=0.01$ ), and trended toward reduced hospital stay and charges.
\end{abstract}

\section{Introduction}

Optimization of antimicrobial therapy in patients with positive blood cultures remains a major challenge. Among hospitalized patients staphylococci are the most frequent bloodstream isolates. Coagulase-negative staphylococci (CoNS) are commonly isolated from blood, but only $\sim 20 \%$ of such cultures represent true infection, the rest being contaminants (Beekmann et al 2005). True CoNS infections are nearly always associated with foreign bodies, particularly vascular catheters and implantable medical devices. Conversely, blood cultures growing Staphylococcus aureus signify bacteremia requiring prompt antibiotic administration and are associated with significant morbidity and mortality. Appropriate use of antibiotics in these settings may improve clinical outcomes, reduce exposure to unnecessary antibiotics, and decrease lengthof-hospitalization and healthcare costs (Dunagan et al 1989).

Furthermore, rapid detection of bloodstream infections and delivery of that information to treating clinicians facilitates optimization of antimicrobial therapy and focused use of healthcare resources (Beekmann et al 2003). Peptide nucleic acid fluorescence in situ hybridization (PNA FISH ${ }^{\mathrm{TM}}$; AdvanDx, Woburn, MA) differentiates S. aureus from CoNS by targeting the $16 \mathrm{~S}$ rRNA of $S$. aureus (Oliveira et al 2002). The test can be performed in 3.5 hours or less and identifies $S$. aureus directly from blood cultures with a sensitivity and specificity exceeding 98\% (Oliveira et al 2003). Use of PNA FISH for differentiation between $S$. aureus and CoNS has been shown to reduce antimicrobial usage and hospital length-of-stay by two days (Forrest et al 2006). PNA FISH has been routinely used in the microbiology laboratory at our institution since 2005. We hypothesized that the addition of a liaison that promptly relays results from the microbiology department to the provider will further improve patient outcomes and utilization of healthcare resources.

Current practice patterns at our hospital are such that there is a direct communication between the laboratory and the clinician with positive gram stains from blood cultures. "Gram-positive cocci in clusters" (GPCC) are relayed to the clinician from the laboratory. However, there is no direct communication between the laboratory and 
healthcare provider with regards to PNA FISH results once they are available (identification of the GPCC as " $\mathrm{S}$. aureus or coagulase-negative staphylococcus"). Clinicians are obligated to follow up with results whenever their time permits and base their decisions accordingly. We propose that an important aspect of a successful early detection program is providing a liaison that can promptly relay results from the microbiology department to the provider and encourage proper therapy, quickly and efficiently. This intervention will likely lead to a decrease in antibiotic misuse, decrease overall hospital costs, and length-of-stay.

To test this hypothesis, we conducted a prospective, randomized controlled study to evaluate whether presence of a liaison that rapidly relays results from laboratory to physicians has any effect on length-of-hospital stay, hospital costs, and antibiotic use.

\section{Methods}

Washington Hospital Center (WHC) is a 907-bed tertiary care medical facility in Washington DC. This hospital's institutional review board approved this study. This was a prospective, randomized, controlled study performed from March 22, 2006 to October 31, 2006.

PNA FISH was performed twice daily on all blood cultures positive for GPCC. Results were entered into the hospital's laboratory information system and a list with the results was faxed twice daily to a laboratory/clinician liaison (LCL). Patients were blindly randomized 1:1, regardless of location within the hospital, to intervention or usual care by alternately assigning patients from the list to each group. Patients whose blood cultures were repeatedly positive for GPCC were only counted once. Patients were excluded if they were younger than 18 years of age, outpatients, or expired prior to the availability of microbiology results. The intervention consisted of notifying the treating clinician of the results and providing general information about the organism isolated within three hours of the test's performance. Patients were further classified according to their location within the hospital at enrollment.

Medical records of enrolled patients were abstracted for demographics, comorbid conditions, location within the hospital, antimicrobial use, length of hospitalization, transfer to intensive care unit (ICU), and mortality.

All baseline patient characteristics were compared by chi-square test except for age and the Charleson Index, which were compared using the t-test. The medians for the outcome variables in the intervention and usual care groups were compared using Wilcoxon rank sum test. Statistical analyses were performed using STATA 9.1 (College station, Texas).

\section{Results}

Two hundred and twenty six patients with blood cultures positive for GPCC were screened. 24 patients were excluded, as they did not meet the inclusion criteria. 202 patients were included in the study and treating clinicians were contacted in 101 of those (intervention). CoNS and S. aureus were identified in 141 and 61 patients, respectively. Among those with CoNS, 72 patients were assigned to the control arm and 69 , to intervention. In those with $S$. aureus 29 were assigned to the control arm and 32, to intervention.

Overall baseline characteristics including demographics, central venous catheters, presence of central line, and concomitant infections were similar in all groups. The mean Charleson comorbidity index was $>3$ and $<5$ for all groups, but was higher in the usual care vs. intervention group for patients with CoNS (4.49 vs.3.71, $\mathrm{p}=0.048$ ).

Rates of mortality, median lengths of hospitalization, and antimicrobial use following enrollment and median total hospital charges are shown in Table 1 for intervention and usualcare groups. Use of the laboratory clinical liaison resulted in reduced overall mortality $(16.8 \%$ vs. $7.9 \% ; p=0.05)$. The biggest impact was seen among patients in the intensive care unit $(47.8 \%$ vs.9.5\%; $\mathrm{p}=0.01)$. Use of the laboratory clinical liaison resulted in a reduction in overall further antimicrobial use $(-2.0$ days, $p=0.01)$ and antimicrobial use in patients with blood cultures showing CoNS $(-2.5$ days, $\mathrm{p}=0.01)$ regardless of location within the hospital. Early notification was associated with a trend toward reduced median hospital charges $(-\$ 19,441.37, p=0.09)$ and for patients with CoNS a decreased subsequent length of hospitalization ( -2 days for all patients and -7 days for ICU patients). Rates of transfer to ICU and utilization of echocardiography were similar in both the intervention and usual care groups.

\section{Discussion}

Rapid differentiation of $S$. aureus from CoNS in blood culture using PNA FISH facilitates focused use of healthcare resources (Forrest et al 2006). However, the clinical impact of rapid diagnostic tests is dependent on expeditious delivery of data to the treating clinician (Doern 1986; Beekmann et al 2003). In patients with bloodstream infections most therapy interventions occur at the time of phlebotomy and after notification of Gram stain results by telephone (Munson et al 2003). In this study we demonstrate that combining a rapid diagnostic test (PNA FISH) with prompt and direct communication of microbiological results is associated with improved outcomes.

Use of the laboratory clinical liaison resulted in reduced overall mortality $(16.8 \%$ vs. $7.9 \% ; p=0.05)$. This 
Table I Outcome variables by organism and location within the hospital: Length of stay and antibiotic use calculated from time of enrollment to hospital discharge. Charges are inclusive of entire hospitalization in United States dollars

\begin{tabular}{|c|c|c|c|c|c|c|c|c|c|}
\hline \multirow[t]{3}{*}{ All hospital locations } & \multicolumn{3}{|l|}{ All patients } & \multicolumn{3}{|l|}{ CoNS } & \multicolumn{3}{|l|}{ S. aureus } \\
\hline & \multirow{2}{*}{$\begin{array}{l}\text { Usual care } \\
(\mathrm{N}=101)\end{array}$} & \multirow{2}{*}{$\begin{array}{l}\text { Notification } \\
(\mathrm{N}=101) \\
\end{array}$} & \multirow[t]{2}{*}{$\mathbf{P}$} & \multirow{2}{*}{$\begin{array}{l}\text { Usual care } \\
(N=72)\end{array}$} & \multirow{2}{*}{$\begin{array}{l}\text { Notification } \\
(\mathrm{N}=69)\end{array}$} & \multirow[t]{2}{*}{$\mathbf{P}$} & \multirow{2}{*}{$\begin{array}{l}\text { Usual care } \\
(\mathrm{N}=29)\end{array}$} & \multirow{2}{*}{$\begin{array}{l}\text { Notification } \\
(\mathrm{N}=32) \\
\end{array}$} & \multirow[t]{2}{*}{$\mathbf{P}$} \\
\hline & & & & & & & & & \\
\hline Hospital stay, median days & 9 & 9 & & 9 & 7 & 0.23 & 14 & 15 & 0.67 \\
\hline Charges, median $\$$ & $92,373.78$ & $72,932.41$ & 0.09 & $65,900.23$ & $64,368.66$ & 0.12 & $110,198.4$ & $103,973.6$ & 0.23 \\
\hline Antibiotics, median day & 3 & 1 & 0.01 & 2.5 & 0 & 0.01 & 9 & 9 & \\
\hline Deaths, No (\%) & $17(16.8)$ & $8(7.9)$ & 0.05 & II (I5.3) & $5(7.2)$ & 0.133 & $6(20.7)$ & $3(9.4)$ & 0.213 \\
\hline Intensive care unit & $(\mathbf{N}=23)$ & $(\mathbf{N}=2 I)$ & & $(N=14)$ & $(N=I I)$ & & $(\mathbf{N}=9)$ & $(10)$ & \\
\hline Hospital stay, median days & 18 & 20 & 0.56 & 18 & 11 & 0.83 & 10.5 & 21.5 & 0.62 \\
\hline $\begin{array}{l}\text { Stay, excluding deaths, } \\
\text { median days }\end{array}$ & 20 & 21 & 0.82 & 18.5 & 13.5 & 0.93 & 61.5 & 22 & 0.22 \\
\hline Charges, median \$ & 245,173 & $|43,63|$ & 0.32 & 308,812 & 195,402 & 0.38 & 233,916 & 142,154 & 0.87 \\
\hline Antibiotics, median day & 5 & 2 & 0.26 & 5 & 0 & 0.02 & 10 & 10 & \\
\hline Deaths, No (\%) & II (47.8) & $2(9.5)$ & 0.01 & $6(42.9)$ & I (9.I) & 0.06 & $5(55.6)$ & $\mathrm{I}(10)$ & 0.03 \\
\hline Nonintensive care unit & $(N=78)$ & $(\mathbf{N}=\mathbf{8 0})$ & & $(N=58)$ & $(\mathbf{N}=58)$ & & $(N=20)$ & $(N=22)$ & \\
\hline Hospital stay, median days & 9 & 8 & 0.3 & 8 & 6.5 & 0.26 & 14 & 11 & 0.50 \\
\hline Charges, median $\$$ & 67,296 & 50,507 & 0.08 & 52,115 & 40,286 & 0.15 & 110,058 & 60,447 & 0.09 \\
\hline Antibiotics, median day & 3 & 1 & 0.02 & 2 & 0 & 0.05 & 8.5 & 3.5 & 0.08 \\
\hline Deaths, No (\%) & $6(7.7)$ & $6(7.5)$ & & $5(8.7)$ & $4(6.9)$ & 0.729 & I (5) & $2(9.1)$ & 0.607 \\
\hline
\end{tabular}

was particularly noticeable in the ICU (47.8\% vs.9.5\%; $\mathrm{p}=0.01)$. Critically ill patients may not tolerate delays in therapy of active bloodstream infections. Receipt of inadequate antimicrobial therapy has been correlated with increased hospital mortality for infected critically ill patients (Kollef et al 1999). We speculate that the reduced mortality associated with our intervention arm was related to early communication with ICU clinicians leading to improved antimicrobial use.

Communication with the treating clinician resulted in a significant reduction in further antimicrobial use overall. This was driven by the significant reduction in antibiotic utilization among patients with CoNS $(-2.5$ days, $\mathrm{p}=0.01)$. Since only a minority of cases with CoNS positive blood cultures represents true bacteremia, early access to microbiological data identifying the blood culture organism as CoNS likely encouraged clinicians to limit further antimicrobial therapy in most of those patients.

False-positive blood cultures have been associated with increased subsequent length of stay and hospital charges (Bates et al 1991). In our patients with CoNS, the intervention was associated with a decreased subsequent length of hospitalization, however, this did not reach statistical significance. Early notification was associated with a trend toward reduced median hospital charges
$(-\$ 19,441.37, p=0.09)$. This is consistent with observations suggesting that time-to-notification significantly impacts hospital charges in bacteremia (Beekmann et al 2003). Enhanced communication of between the laboratory and clinicians likely facilitated more rapid utilization of focused therapy.

Baseline comorbidities were compared using the Charlson comorbidity index, a weighted score that takes into account the number and the seriousness of comorbid diseases. Oneyear mortality has been reported as $52 \%$ for patients with score of $3-4$ and $85 \%$ with a score of 5 or more (Charlson et al 1987). Mean index scores for all groups in our study were $>3$ and $<5$. A possible confounder for our study was a higher mean index for control patients with CoNS compared with the intervention group (4.49 vs.3.71, $\mathrm{p}=0.048$ ). An index of 3 predicts higher mortality for patients with S. aureus bacteremia (Lesens et al 2003). We do not know whether a difference in Charlson index above this threshold impacts the outcomes of hospitalized patients with CoNS bacteremia and pseudobacteremia.

In conclusion, this study suggests that in a clinical scenario where rapid access to laboratory data can significantly alter the course of treatment, delivery of that data via a laboratory clinician liaison can improve clinical outcomes and utilization of resources. 


\section{References}

Bates DW, Goldman L, Lee TH. 1991. Contaminant blood cultures and resource utilization. The true consequences of false-positive results. JAMA, 265:365-9.

Beekmann SE, Diekema DJ, Doern GV. 2005. Determining the clinical significance of coagulase-negative staphylococci isolated from blood cultures. Infect Control Hosp Epidemiol, 26:559-66.

Beekmann SE, Diekema DJ, Chapin KC, et al. 2003. Effects of rapid detection of bloodstream infections on length of hospitalization and hospital charges. J Clin Microbiol, 41:3119-25.

Charlson ME, Pompei P, Ales KL, et al. 1987. A new method of classifying prognostic comorbidity in longitudinal studies: development and validation. $J$ Chronic Dis, 40:373-83.

Doern GV. 1986. Clinically expedient reporting of rapid diagnostic test information. Diagn Microbiol Infect Dis, 4:151S-156S.

Dunagan WC, Woodward RS, Medoff G, et al. 1989. Antimicrobial misuse in patients with positive blood cultures. Am J Med, 87:253-9.

Forrest GN, Mehta S, Weekes E, et al. 2006. Impact of rapid in situ hybridization testing on coagulase-negative staphylococci positive blood cultures. J Antimicrob Chemother, 58:154-8.
Kollef MH, Sherman G, Ward S, et al. 1999. Inadequate antimicrobial treatment of infections: a risk factor for hospital mortality among critically ill patients. Chest, 115:462-74.

Lesens O, Methlin C, Hansmann Y, et al. 2003. Role of comorbidity in mortality related to Staphylococcus aureus bacteremia: a prospective study using the Charlson weighted index of comorbidity. Infect Control Hosp Epidemiol, 24:890-6

Munson EL, Diekema DJ, Beekmann SE, et al. 2003. Detection and treatment of bloodstream infection: laboratory reporting and antimicrobial management. J Clin Microbiol, 41:495-7.

Oliveira K, Brecher SM, Durbin A, et al. 2003. Direct identification of Staphylococcus aureus from positive blood culture bottles. J Clin Microbiol, 41:889-91.

Oliveira K, Procop GW, Wilson D, et al. 2002. Rapid identification of Staphylococcus aureus directly from blood cultures by fluorescence in situ hybridization with peptide nucleic acid probes. J Clin Microbiol, 40:247-51. 